\title{
Visualization of a Flow around the Surface with Different Roughness Coefficients
}

\author{
Win Thu ${ }^{1}$, D. V. Ilin² ${ }^{2}$ N. M. Skornyakova 3 \\ National research University Moscow power engineering Institute "MPEI" \\ 1. ORCID: 0000-0002-0459-784X, winthu.thu51@gmail.com \\ 2 ORCID: 0000-0001-6374-1162, d-v-ilin@yandex.ru \\ 3 ORCID: 0000-0002-2919-6428, nmskorn@mail.ru
}

\begin{abstract}
The work purpose is to determine the impact of a rough surface on the airflow around it, depending on the degree of the surface roughness.

The experimental research of the process of air stream flowing around samples of flat and rough surfaces has been performed. The experimental results have been compared with theoretical ones obtained by executing a simulation of the considered process which is based on RANS equations.

Keywords: particle image velocimetry, aerodynamics, flow around surfaces, rough surface, roughness degree, flow simulation.
\end{abstract}

\section{Introduction}

Gas and hydrodynamic flows are present in many technical devices and machines, in various scientific setups. Such phenomena occur, for example, when air flows around transport vehicles in motion or when the ship's screw engines are operating in water environment. The variety of similar effects has led to the emergence of many methods for researching the patterns of vortex distribution and turbulent flows in different kinds of objects. Flow spread conditions can be quite harsh: high temperature (thousands of degrees) or high speeds (units of $\mathrm{km} / \mathrm{s}$ ), therefore contactless optical methods which don't introduce any disturbances into investigated process gain an advantage. So, one of these methods is particle image velocimetry (PIV) [1].

Particle image velocimetry is applied to measure the velocity in the selected twodimensional section or three-dimensional flow volume in hydro- and aerodynamic experiments [2 - 4]. The method allows to register the field of the instantaneous spatial distribution of velocities. PIV is used, for example, to measure the speeds of gas turbine and aircraft engines, to optimize the flow around aircraft [5 - 10]. It has a number of advantages, such as the absence of a disturbing effect on the flow and a wide dynamic range of measured velocities [11-13].

To date, PIV is widely used and in demand in various technical fields, such as automobile and aircraft construction, power and mechanical engineering. The method is also applied in a variety of scientific fields: for the study of phenomena in ecology and meteorology. For example, PIV is used to model processes of pollution of the ocean coastal zone by wastewater discharge systems, to measure parameters of the air flow over the agitated surface [14], to investigate flow characteristics in the nozzle of a gas turbine engine [15].

The rapid development of laser technology, electronics, and recording equipment contributes in every possible way to the improvement of experimental methods, allowing to measure instantaneous velocity fields with higher spatial and temporal resolution and to automate the processing. The PIV method is currently widely used in various fields of science and production, the boundaries of its application are constantly expanding. However at the 
moment its applicability to the study of the aerodynamics of combustion chambers and boilers hasn't been investigated. In these objects, a special fuel-air mixture is fed into a furnace and burns inside the chamber.

The research purpose is to determine the impact of surface roughness on the air flowing around this surface. As a result of this study, the dependence of changes occurring in air stream during the flowing of surfaces with different degrees of roughness, depending on characteristic dimensions of irregularities of this surface, can be revealed.

\section{Research method}

To measure the instantaneous flow rate, LaVision FlowMaster PIV flow diagnostics system was used. It consisted of a pulsed Nd:YAG laser, operating at wavelength of $532 \mathrm{~nm}$ (repetition frequency of pulses pairs is $6 \mathrm{~Hz}$, pulse duration is 6-9 ns with pulse energy of 150 $\mathrm{MJ}$, delay between pulses in a pair is $0.01 \mathrm{~s}$ ). A CCD camera Imager pro SX $5 \mathrm{M}$ (resolution of $5 \mathrm{MP}$, bit depth of 12 bits) and a syncing processor acted as a radiation receiver. System control, data collection, storage and processing were carried out using a computer with DaVis software. The aerosol flow (average diameter of 1 microns) was illuminated by a laser plane with a thickness of $3 \mathrm{~mm}$. During recording images of particles scattering laser radiation, an optical filter was used.

\section{Experimental setup}

To ensure the possibility of performing studies of air flow around a rough surface, an experimental setup was developed (Figure 1). This installation allows using particle image velocimetry to study flow around surfaces. A sample of the surface (3) is placed in a plexiglass cube. An aerosol created by aerosol generator (5) is launched into the cube. A laser plane (2) is formed by special optical system, which highlights particles in the required cross-section. The air flow is created using an industrial hair dryer. The stream flows around the surface in the direction along the laser plane. Images of the stream are recorded by the digital video camera (4). The resulting images are transmitted to the computer, where they are processed. As a result of processing, vector fields of flow velocity can be obtained.

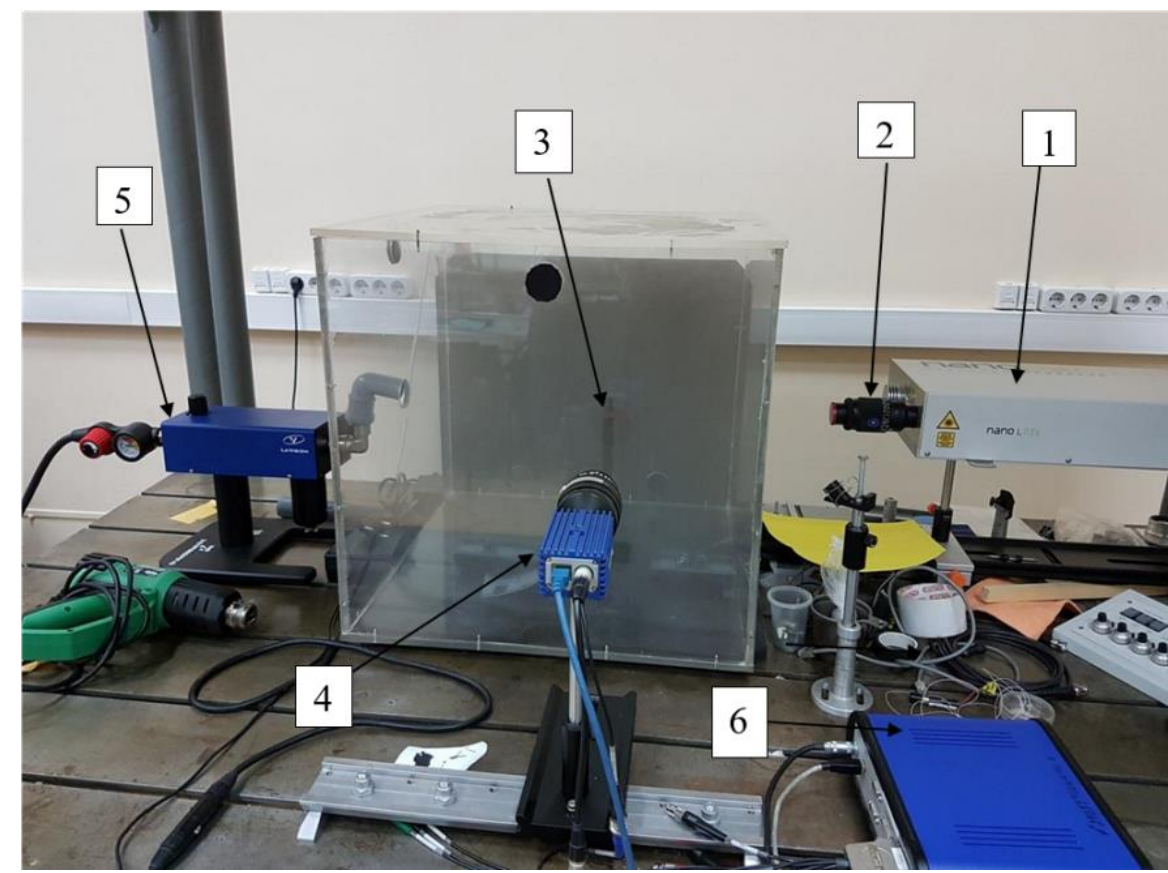

1 - laser, 2 - optical system, 3 - sample of surface, 4 - digital camera, 5 - aerosol generator, 6 - syncing processor

Fig. 1. Scheme of experimental setup 
The obtained images were subjected to cross-correlation processing in LAVISION DaVis program [16]. The main processing parameters on which the visualization result depends are the size and shape of the survey window, the overlap percentage of the surveyed areas. As a result, vector velocity fields of the flow have been obtained, and the average velocity profiles in the selected plane have been calculated.

\section{Experimental results}

As a result of the experiments, images of the air flow around the samples with various degrees of roughness have been obtained. An example of such image is shown in Figure 2.

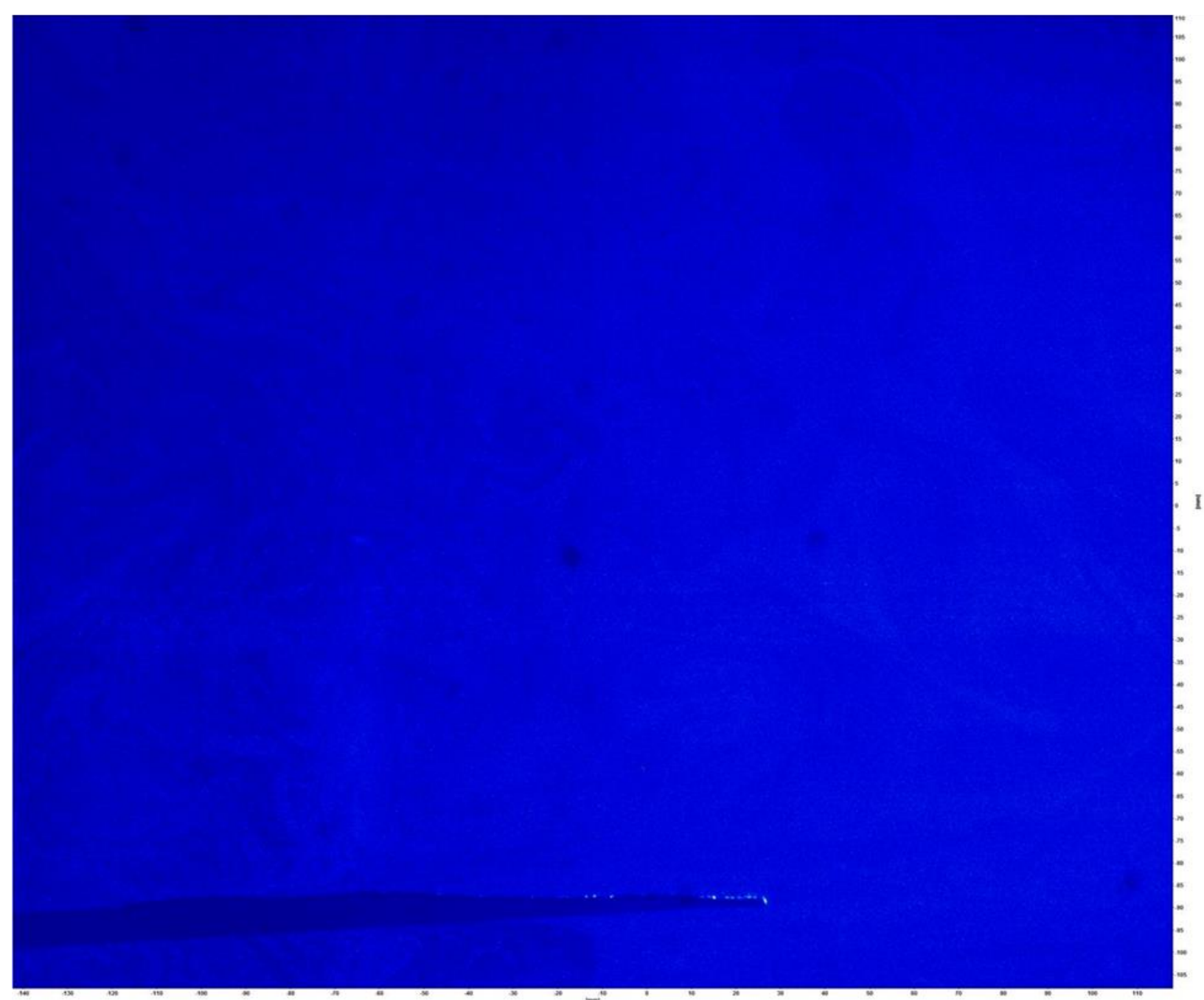

Fig. 2. Example of experimental flow image

For each value of the flow rate five series of flow images were recorded, each of which had ten double frames. As a result of cross-correlation processing vector fields of the flow velocity were obtained in the section, which has been located along the flow in a plane normal to the sample surface. The processing was performed with the following parameters: the standard cross-correlation method, the size of the survey area was 64 x 64 pixels, the overlap was 50\%.

Then, for each series of measurements, the obtained vector fields were averaged. So the magnitude and direction of the resulting vector were averaged for each survey area. This made it possible to significantly reduce the influence of random emissions caused mainly by flow vortices that occurred during the formation of the flow by an industrial hair dryer.

The averaged vector fields of the flow velocity for a rough surface sample, which is a fragment of P1Oo sandpaper, at different values of the flow velocity are presented in Figures 3 -7 . 


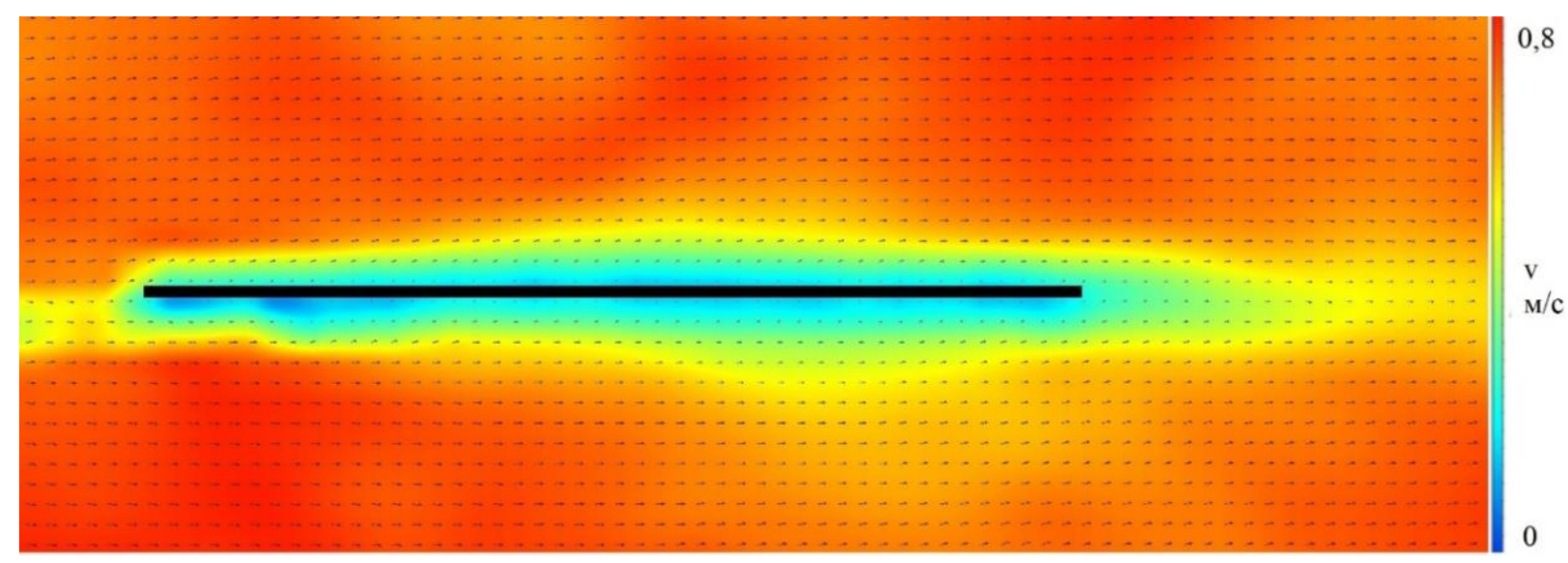

Fig. 3. Vector field for sample P100 at flow velocity 1

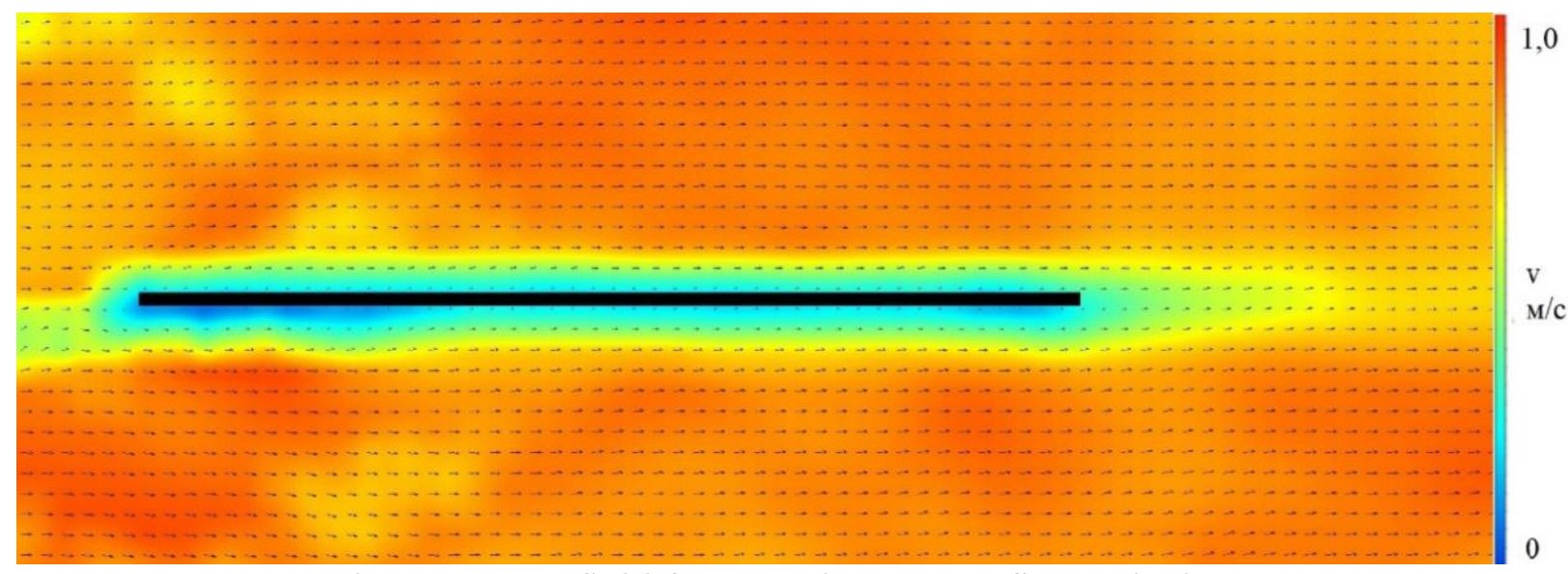

Fig. 4. Vector field for sample P100 at flow velocity 2

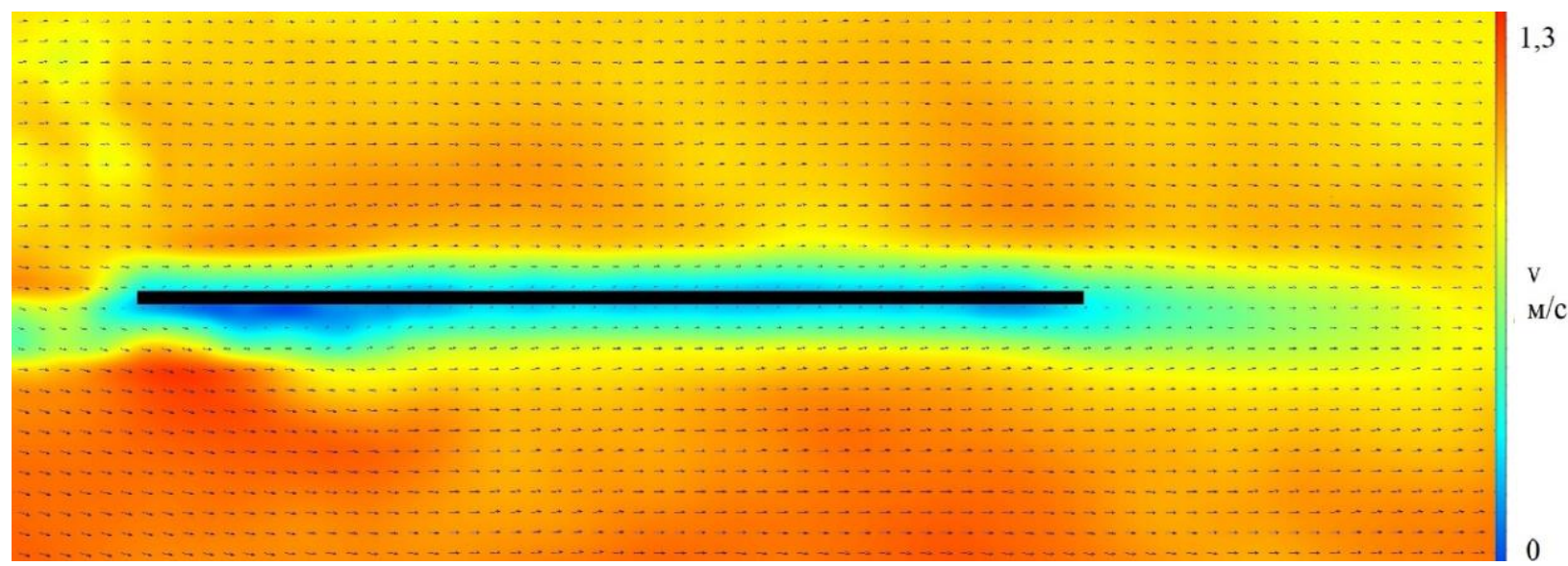

Fig. 5. Vector field for sample P100 at flow velocity 3 


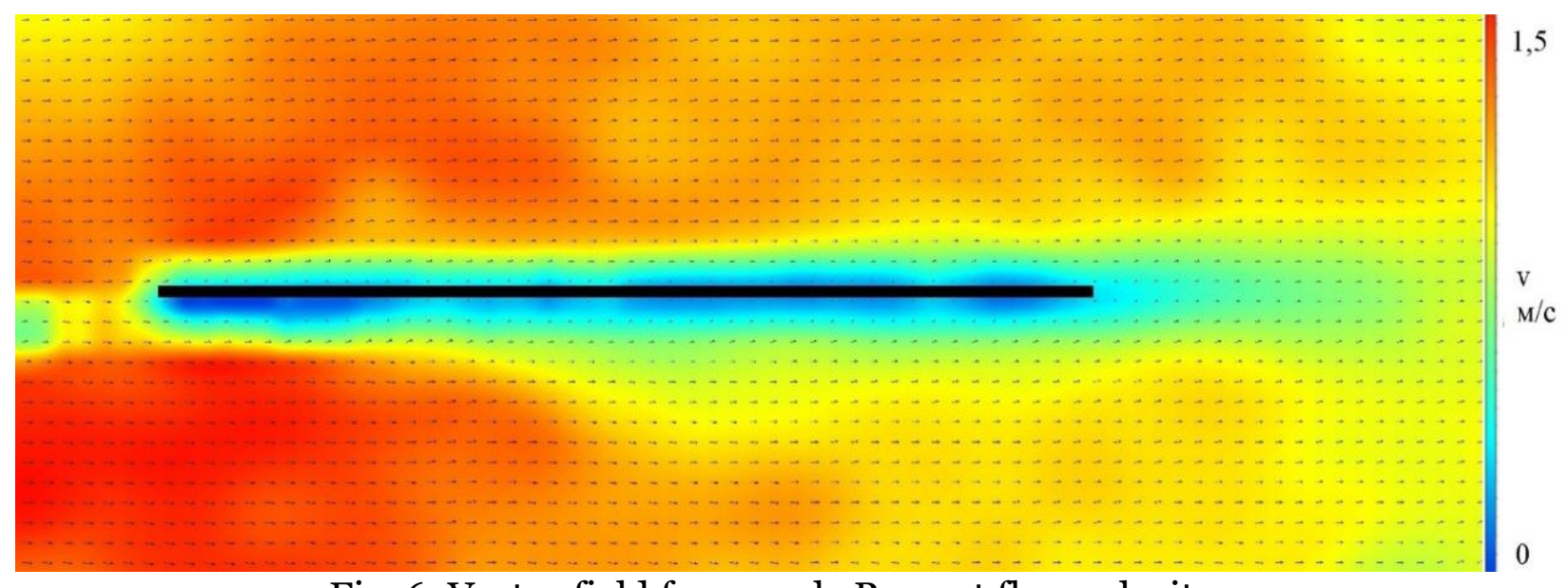

Fig. 6. Vector field for sample P100 at flow velocity 4

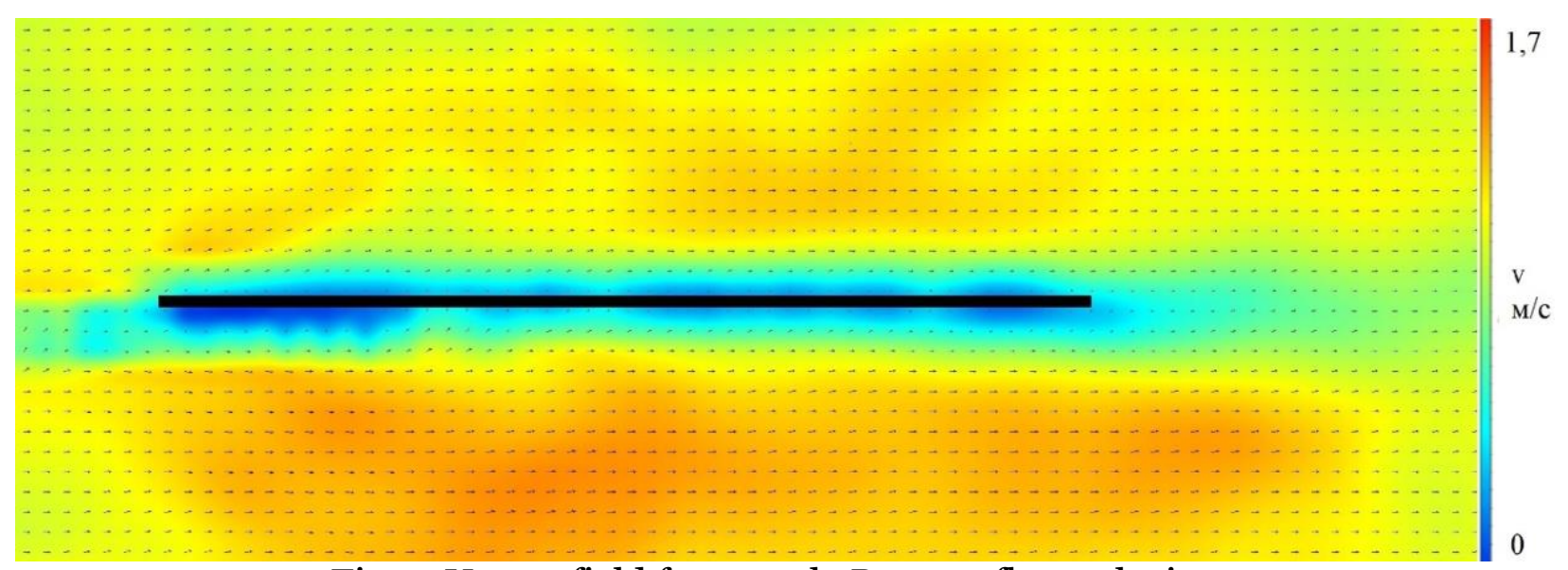

Fig. 7. Vector field for sample P100 at flow velocity 5

The comparison of the flow velocity vector fields obtained experimentally with the flow velocity distributions obtained as a result of modeling can be carried out mainly visually. Such a comparison is of an exclusively qualitative nature. Therefore additional processing of the obtained data is required in order to move from qualitative to quantitative analysis.

In order to perform a quantitative comparison of the experimental results with the simulation results, plots of the flow velocity dependence over the sample surface on the longitudinal coordinate has been constructed. Then these graphs were averaged. As a result, one plot was obtained for each velocity value, which took into account all measurements made at the appropriate flow velocity.

Experimental results for a case of a smooth surface flow were processed in a similar way. Vector fields of the flow velocity obtained as a result of cross-correlation processing for the sample of the smooth surface at different values of the flow velocity are shown in Figures 8 12. 


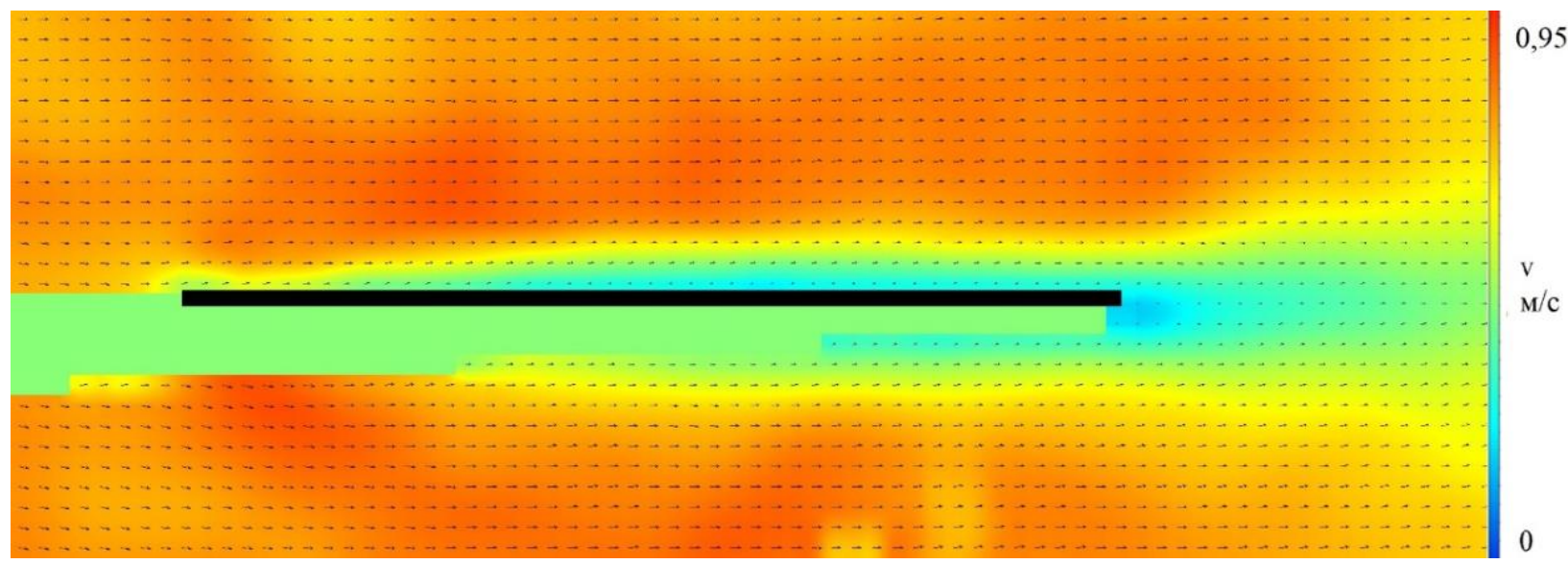

Fig. 8. Vector field for sample of smooth surface at flow velocity 1

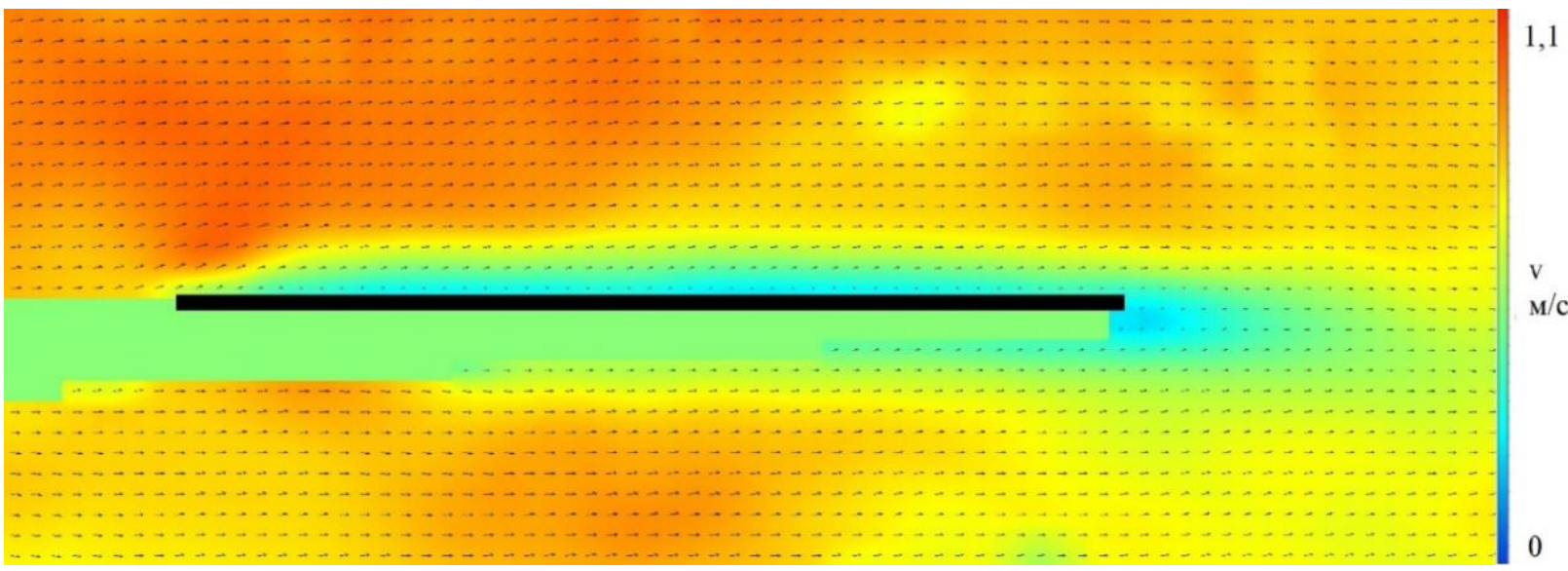

Fig. 9. Vector field for sample of smooth surface at flow velocity 2

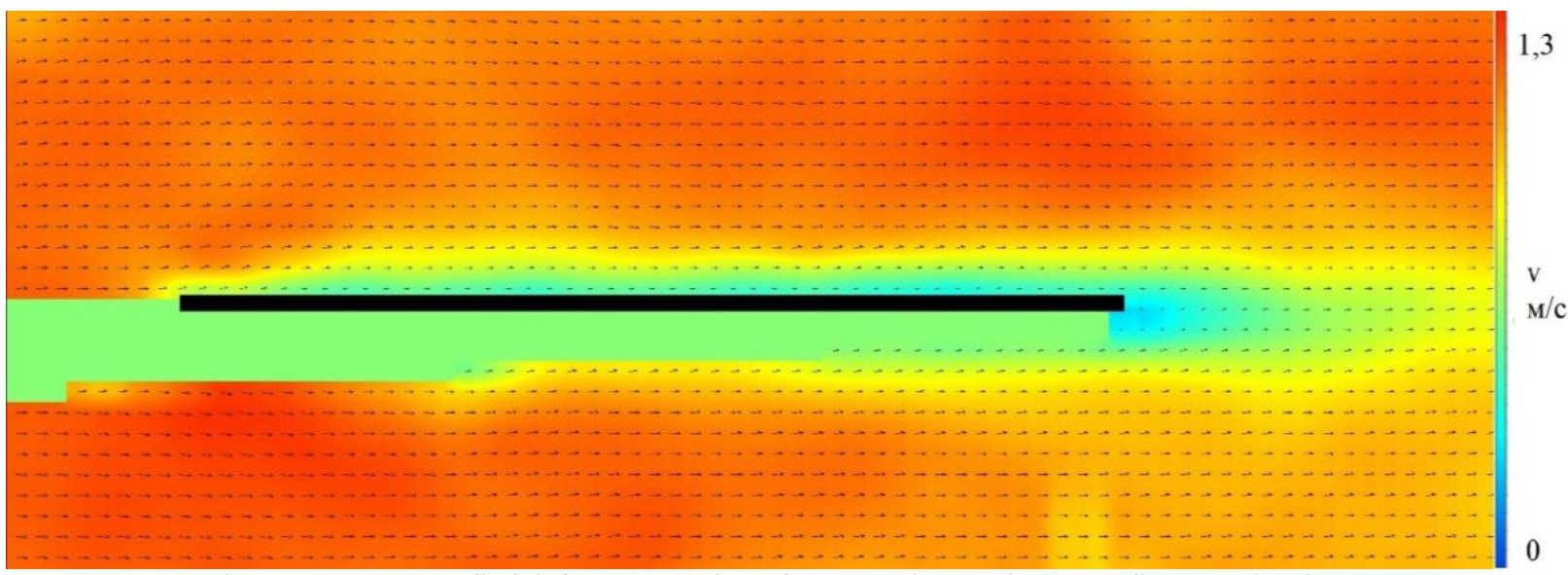

Fig. 10. Vector field for sample of smooth surface at flow velocity 3 


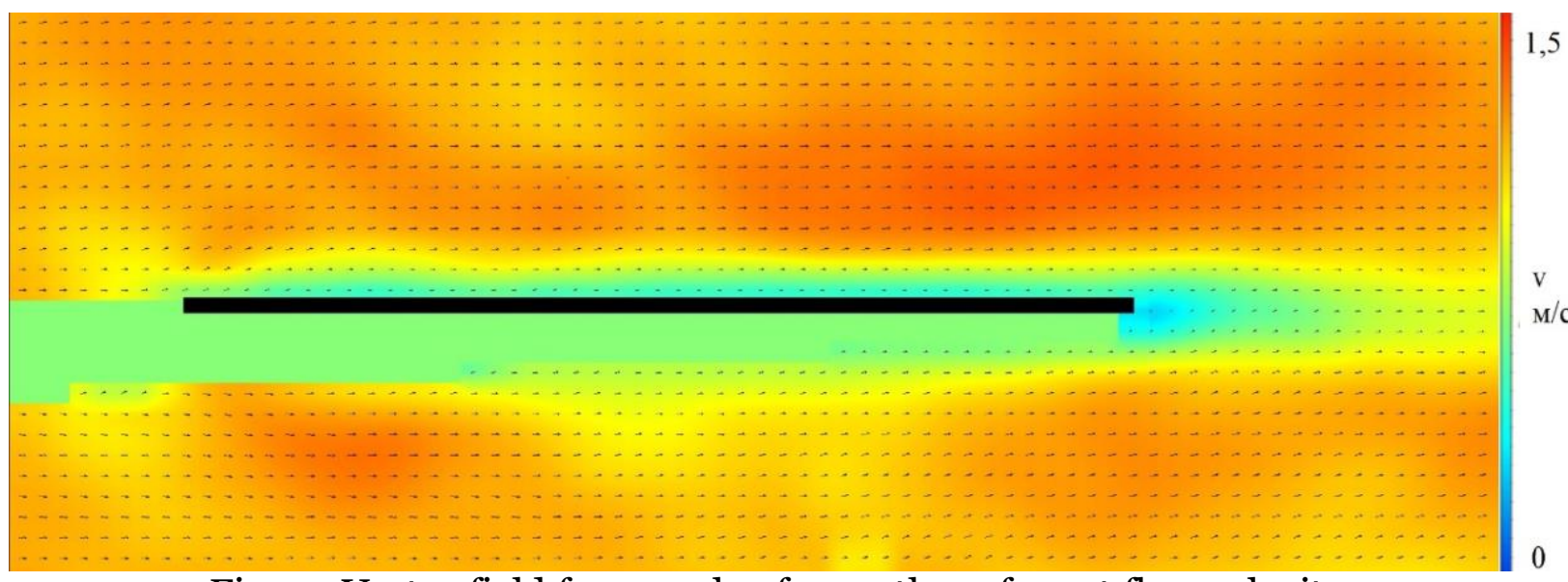

Fig. 11. Vector field for sample of smooth surface at flow velocity 4

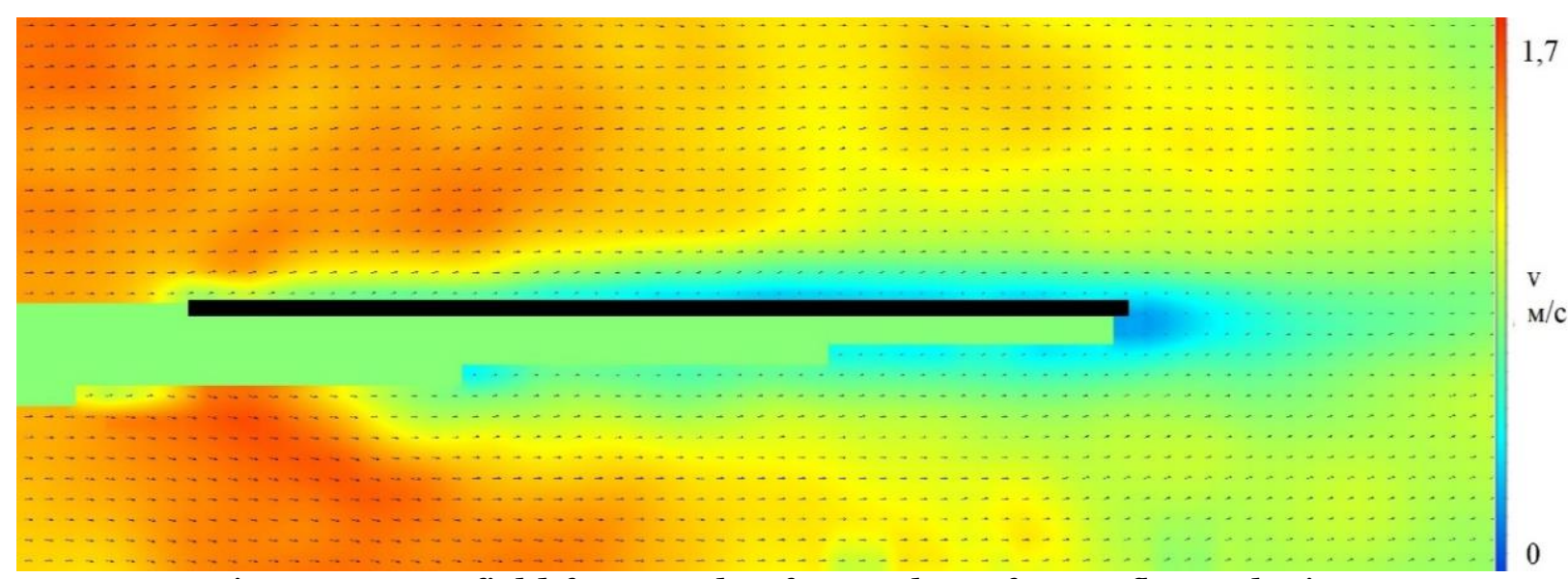

Fig. 12. Vector field for sample of smooth surface at flow velocity 5

\section{Comparison of experimental and theoretical results}

\subsection{Rough surfaces}

For comparison, all the plots obtained experimentally and as a result of modeling were transferred in pairs to one coordinate system. At the same time, the approximation of the obtained plots was carried out, which made it possible to reduce the influence of random outliers in data arrays on the resulting plots. Comparative plots for the rough surface sample P100 at flow velocity 1-5 are shown in Figure 13.

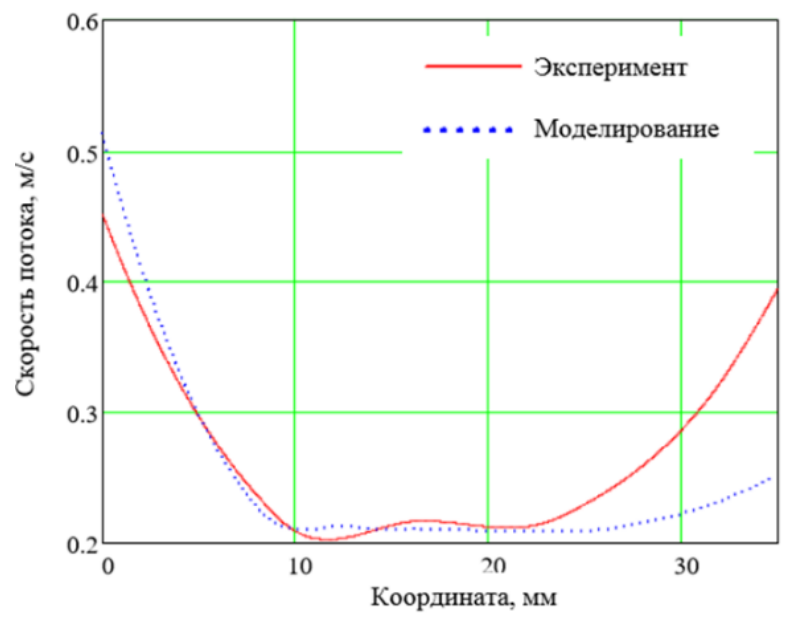

(a)

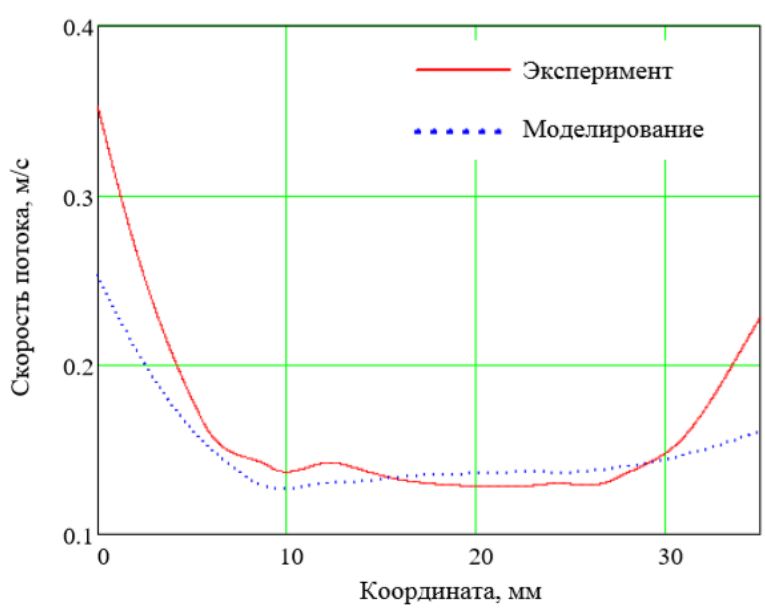

(b) 


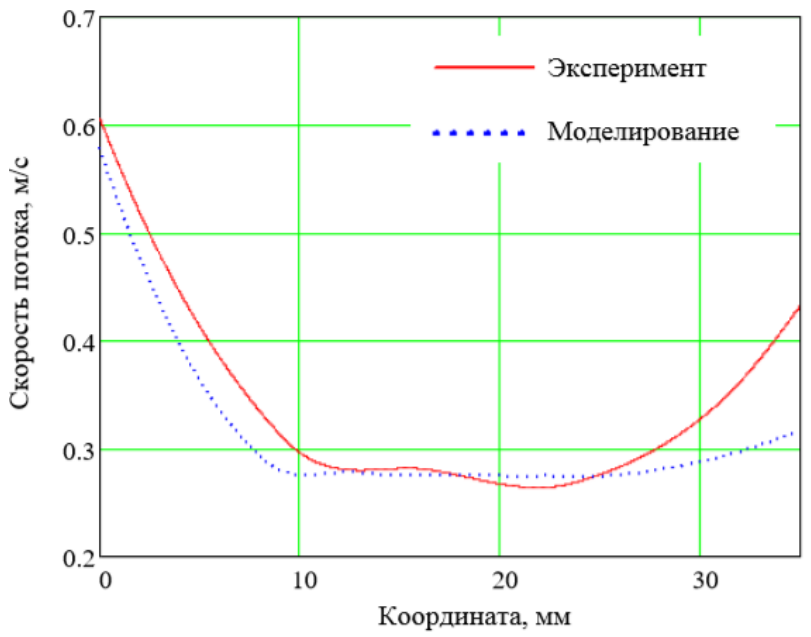

(c)

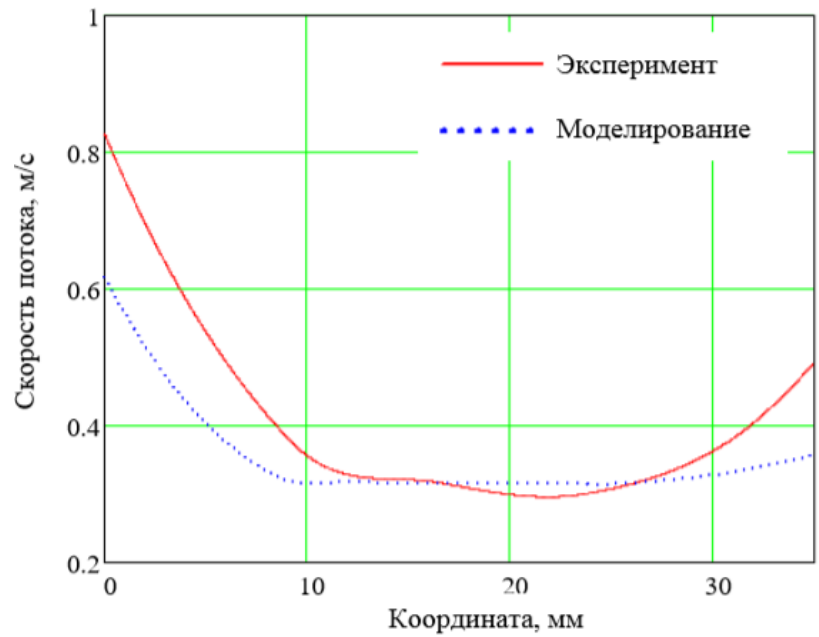

(d)

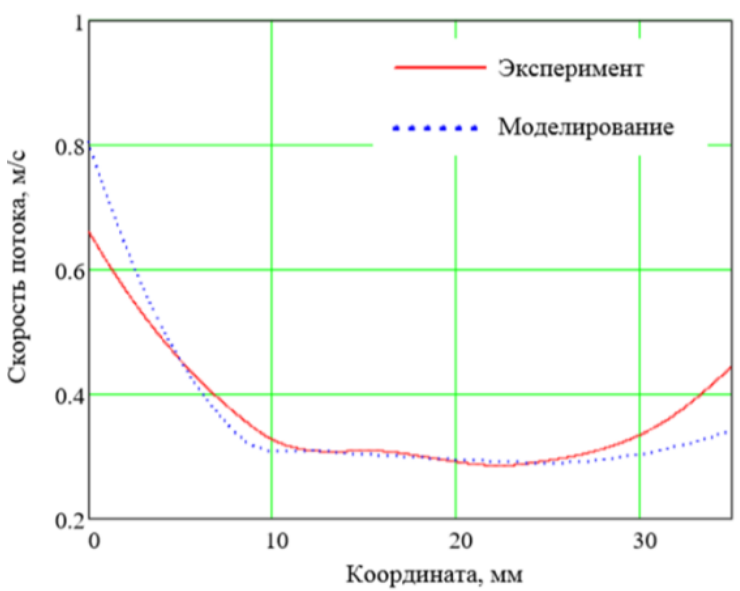

(e)

Fig.13. Dependence of flow velocity over the surface of the sample P100 at flow velocities 1 (a), 2 (b), 3 (c), 4 (d), 5 (e)

In this case, theoretical results were obtained by modeling the flow around the rough surface with some simplifications. First, the surface model in the initial version was set in a significantly simplified form, in which it had noticeable structural differences from the real sample of the rough surface made of sandpaper. Secondly, to simplify and, accordingly, to speed up the modeling process, only a part of the air flow was considered, without taking into account the fact that the samples used in the experiments had a certain thickness, which also affected the results obtained. This revealed the need to improve the parameters of the surface models and the air flow used in modeling.

\subsection{Smooth surface}

Comparative plots for the case of flow around the sample of the smooth surface were obtained in a similar way, by transferring into a single coordinate system experimental plots of the flow velocity dependence modeling plots. These plots are presented in Figure 14. 


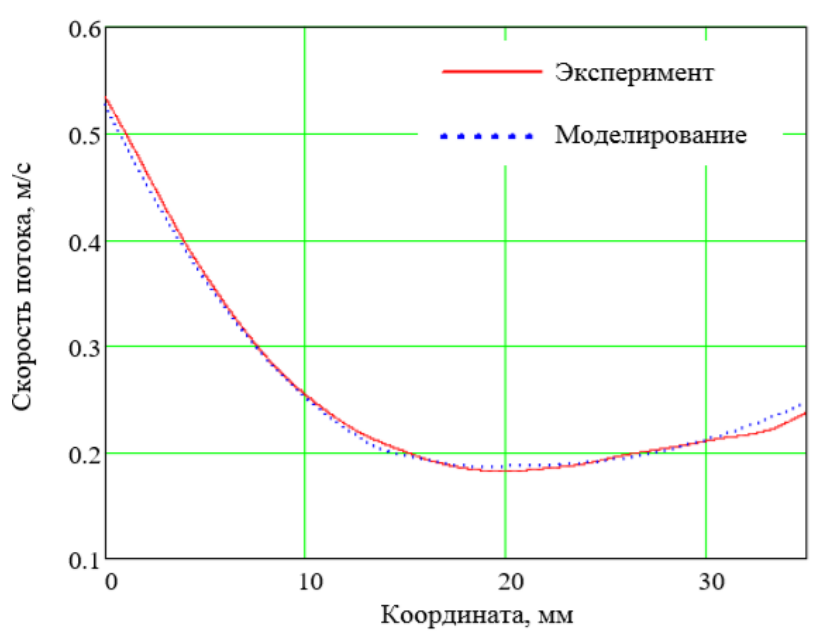

(a)

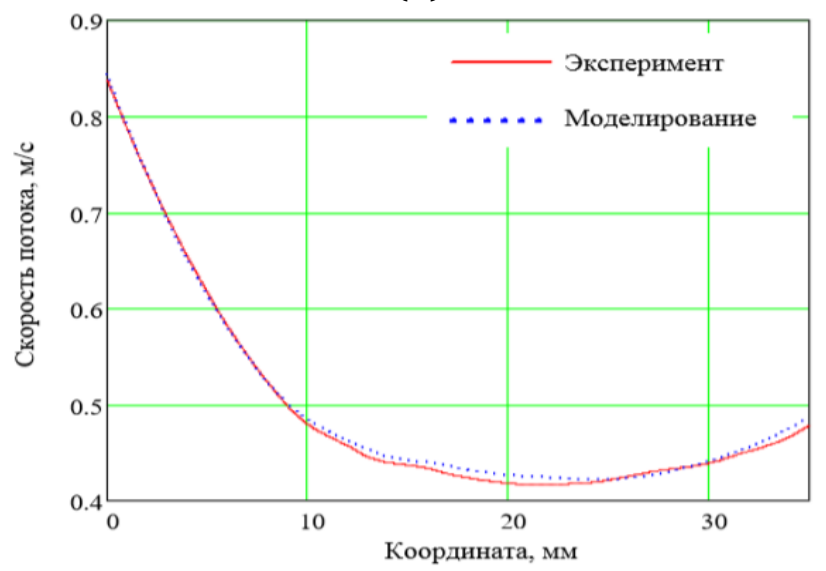

(c)

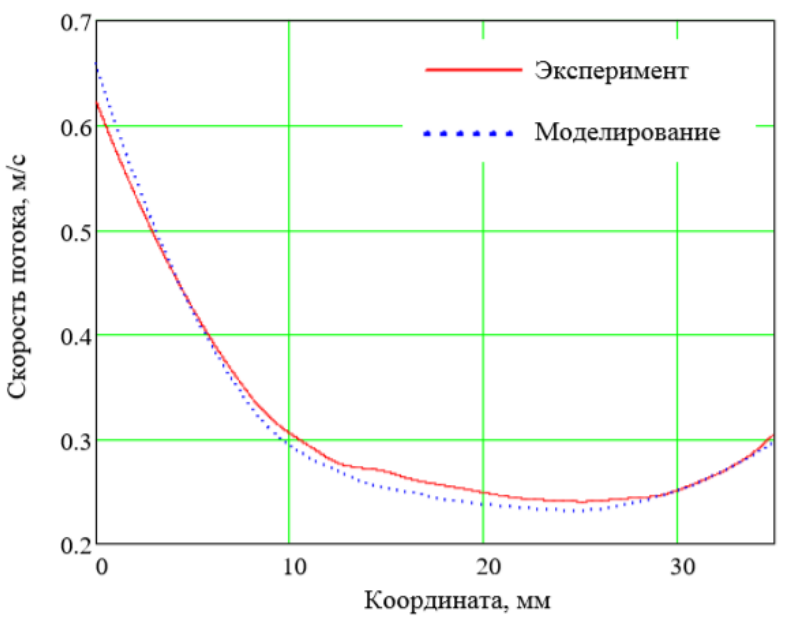

(b)

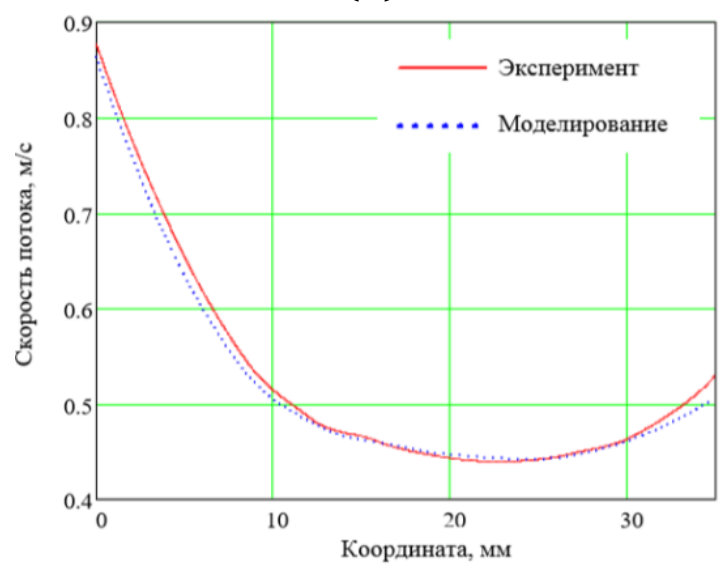

(d)

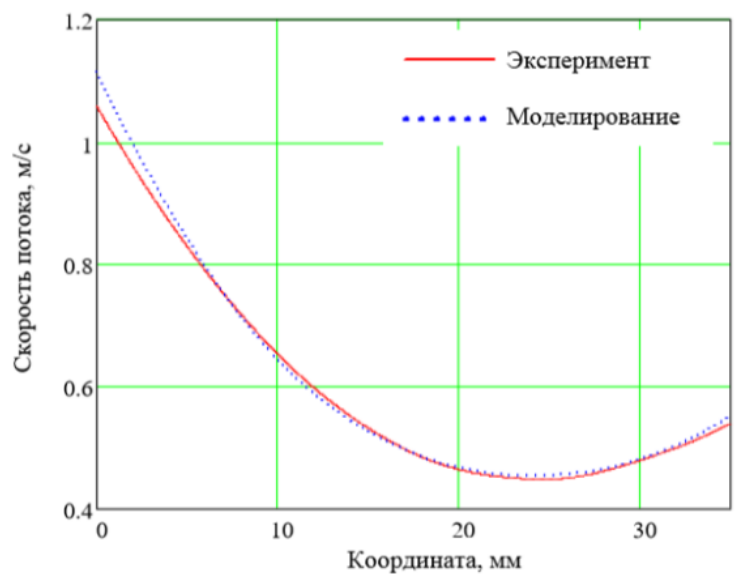

(e)

Fig.14. Dependence of flow velocity over the smooth surface at flow velocities 1 (a), 2 (b), 3 (c), 4 (d), 5 (e)

\section{Conclusion}

During the research, the process of air flow around surfaces with different degrees of roughness has been studied. The phenomenon was investigated both experimentally and theoretically, which consisted of physical and mathematical simulations of the process.

In order to perform aerodynamic studies using PIV method the experimental setup has been developed. As the experiments results have shown, the setup allows us to successfully carry out such experimental studies. However, to increase the accuracy of the experimental results, it is desirable to use another method of creating an air flow, in which the generated flow will be as close as possible to the laminar in nature. 
The result of the experimental part was to obtain vector flow velocity fields for samples of the smooth surface and four rough surfaces with different degrees of roughness at different values of the flow speed. From the obtained vector fields, information was extracted about the flow velocity over the surface of each of the samples, depending on the coordinate along the flow.

In order to verify the experimental data mathematical modeling of the process has been performed. The modeling results are spatial distributions of the flow velocity in the cross section normal to the sample surface, which are similar to the experimentally obtained vector fields for visual comparison of flows, as well as similar dependences of the flow velocity on the coordinate along the flow. When modeling the flow around rough surfaces, a problem arose related to the need to use large computing power, because the amount of required calculations depends on the surface parameters. This difficulty lies in the fact that with a decrease in the degree of surface roughness, the average characteristic dimensions of surface irregularities become smaller, and their number on the sample section of the same length, respectively, increases. So creating sufficiently realistic models of rough surfaces inevitably leads to a significant increase in the time spent on calculations.

\section{Acknowledgments}

This work was funded by Russian Foundation for Basic Research (project 19-07-00921A).

\section{References}

1. S. Y. Kolesnikov, S. V. Bayun, N. M. Skornyakova, N. L. Ryazanova. Investigation of Laminar flow in a fine aerosol by the AICH method // the thirteenth international scientific and technical conference "Optical methods of flow research". Moscow, June 29 - July 03, 2015 - p. $518-525$.

2. Adrian R.J. Twenty years of particle image velocimetry // Experiments in Fluids, 2005, no. 39 (2), pp. $159-169$

3. Raffel M., Willert C., Wereley S., Kompenhans J. Particle image velocimetry: a practical guide, New York, Springer, 2007, 448 p.

4. Adrian R.J., Westerweel J. Particle Image Velocimetry, Cambridge, UK, Cambridge University Press, 2010, 558 p.

5. Zakharov D. L. Development of methods for measuring velocity and concentration fields using the PIV method in flows characteristic of gas turbine engines // Proceedings of the MAI. No. 45, series of 2011 page address: http://trudymai.ru/published.php?ID=25391

6. Kostarev K. G., Batalov V. G., Mizev A. I., Sukhanovsky A. N., Shmyrov A.V. Hydrodynamic aspects of the processes of formation and decay of the fuel film created by the nozzle of the combustion chamber of an aircraft engine // Bulletin of the Perm scientific center of the URAL branch of the Russian Academy of Sciences, 2017, no. 1, pp. 52-56.

7. Znamenskaya I., Glazyrin F., Koroteeva E., and Naumov D. PIV investigation of lowpressure pulse discharge flow // 10th Pacific Symposium on Flow Visualization and Image Processing (PSFVIP-10), University of Naples Federico II, Italy Naples, Italy, 2015 pp. 153-1$153-5$.

8. Markovich D.M., Abdurakipov S.S., Chikishev L.M., Dulin V.M., Hanjalic K. Comparative analysis of low-and high-swirl confined flames and jets by proper orthogonal and dynamic mode decompositions // Physics of Fluids, 2014, vol. 26, no. 6, pp. 65109.

9. Batalov V. G., Stepanov R. A., Sukhanovsky A. N. application of direct optical measurement methods to study the characteristics of a two-phase flow // Proceedings of the MAI. 2014 № 76 URL: http://trudymai.ru/published.php?ID=50074

10. Orlov A.V., Brazhnikov M. Yu., Levchenko A. A. Formation of a large-scale coherent vortex in two-dimensional turbulence // experimental and theoretical physics. 2018 Vol. 107 No. 3-4. Pp. $166-171$. 
11. We briefly L. M., Dulin V. M., Lobasov A. S., Markovich D. M. influence Of large-scale vortical structures in the flame shape in the flow of a swirling jet // Combustion and explosion. 2018 Vol. 11 No. 2 P. $31-39$.

12. Znamenskaya I. A., Glazyrin F. N., doroshchenko I. A. and others. Digital anemometry based on particle images as applied to shock-wave flows // III industry conference on measurement technology and Metrology for aircraft research, KIMILA - 2018 Proceedings (Zhukovsky, June 05-06, 2018). - The Zhukovsky Central Aerohydrodynamic Institute. Professor N.E. Zhukovsky, 2018 P. $292-301$.

13. Voitkov I. S., Kuznetsov G. V., Strizhak P. A. Determination of the temperature of gases during the passage of water aerosol through them // Letters to the journal of technical physics.2017 Vol. 43 No. 6 P. 48-55.

14. Sergeev D. A. Use of modern methods of anemometry based on particle images (pivmethods) in laboratory modeling of geophysical flows// Bulletin of the Lobachevsky University of Nizhny Novgorod, 2011, no. 4 (2), pp. 522-524.

15. Batalov V. G. Stepanov R. A., Sukhanovsky A. N. Application of direct optical methods for studying the characteristics of a two-phase flow// electronic journal "Proceedings of MAI". Issue № 76

16. Raffel, M., Willert, C.E., Wereley, S.T., Kompenhans, J. Particle image velocimetry: a practical guide/ 2nd ed. 2007, XX, P. 79-95. 